\title{
Guar gum and similar soluble fibers in the regulation of cholesterol metabolism: Current understandings and future research priorities
}

\author{
Todd C Rideout ${ }^{\prime}$ \\ Scott V Harding' \\ Peter JH Jones' \\ Ming Z Fan ${ }^{2}$ \\ 'Richardson Centre for Functional \\ Foods and Nutraceuticals, University \\ of Manitoba, Winnipeg, Manitoba, \\ Canada; ${ }^{2}$ Centre for Nutrition \\ Modeling, Department of Animal \\ and Poultry Science, University \\ of Guelph, Guelph, Ontario, Canada
}

Correspondence: Todd C Rideout Richardson Centre for Functional Foods and Nutraceuticals, University of Manitoba, 196 Innovation Drive Winnipeg, MB R3T 6C5, Canada

Tel +l 2044748383

Fax +I 2044747552

Email_trideout@umanitoba.ca

\begin{abstract}
The hypocholesterolemic effects associated with soluble fiber consumption are clear from animal model and human clinical investigations. Moreover, the modulation of whole-body cholesterol metabolism in response to dietary fiber consumption, including intestinal cholesterol absorption and fecal sterol and bile acid loss, has been the subject of many published reports. However, our understanding of how dietary fibers regulate molecular events at the gene/protein level and alter cellular cholesterol metabolism is limited. The modern emphasis on molecular nutrition and rapid progress in 'high-dimensional' biological techniques will permit further explorations of the role of genetic polymorphisms in determining the variable interindividual responses to soluble fibers. Furthermore, with traditional molecular biology tools and the application of 'omic' technology, specific insight into how fibers modulate the expression of genes and proteins that regulate intestinal cholesterol absorption and alter hepatic sterol balance will be gained. Detailed knowledge of the molecular mechanisms by which soluble fibers reduce plasma cholesterol concentrations is paramount to developing novel fiber-based "cocktails" that target specific metabolic pathways to gain maximal cholesterol reductions.
\end{abstract}

Keywords: dietary fiber, cholesterol, bile acids, gene, protein

\section{Introduction}

Dietary fiber may be considered the 'dinosaur' of functional foods. The hypolipidemic effects and cardioprotective benefits associated with dietary soluble fiber consumption are abundantly clear from the results of human clinical trials (Anderson et al 1984, 1991, 2000b; Bell et al 1989; Braaten et al 1994; Naumann et al 2006), animal feeding studies (van Bennekum et al 2005; Venkatesan et al 2007), epidemiological investigations (Lairon et al 2005), and meta-analysis reports (Ripsin et al 1992; Anderson et al 2000a; Anderson and Major 2002; Castro et al 2005). Furthermore, the mechanisms by which soluble fibers modulate cholesterol metabolism and effectively reduce circulating cholesterol concentrations have been the subject of many published reports (Fernandez et al 1999; Queenan et al 2007). Such an extensive research history begs the question: Is the fiber story over? Advancements in any biological discipline often require an assessment of current knowledge and a reflection on what is not understood or has yet to be pursued. The objective of this review is to assess what is known regarding the role of soluble dietary fiber in modulating cholesterol metabolism and reducing cardiovascular disease risk. To contextualize the place of fiber in modern human health and disease prevention, we will begin with a brief account of the history of dietary fiber research and the modern dietary fiber classification system. An additional objective of this review article is to explore what is known regarding the 
mechanisms by which fiber consumption is thought to reduce plasma cholesterol concentrations. Finally, with the modern emphasis on molecular nutrition and the application of 'highdimensional biology' or 'omic' technology, we will review recent progress in understanding how fiber consumption may regulate the enterohepatic metabolism of cholesterol at the molecular level and highlight current research priorities that will advance our existing knowledge base.

\section{A brief history of dietary fiber}

In the mid 1970s, as the controversy surrounding the role of cholesterol in atherosclerosis reached an unparalleled point of contention within the medical community (Steinberg 2004, 2005), the dietary fiber hypothesis emerged as the new paradigm of nutrition in human health and disease prevention. While modern dietary fiber nutrition stemmed from observational and epidemiological studies in the 1970s, the hypothesis that the consumption of coarse foods of plant origin could modulate human health was not an entirely new concept. As early as $430 \mathrm{BC}$, Hippocrates understood the link between dietary fiber and the 'diseases which befall a man' and recognized that 'to the human body it makes a great difference whether the bread be fine or coarse; of wheat with or without the hull' (Adams 1939). Indeed, advances in the analysis (McCance et al 1936) and energy value (Atwater 1900) of these 'indigestible carbohydrates' had already been made by the 1930s. However, during the first half of the 20th century, published reports on the functionality of dietary fiber were few and the idea that a group of indigestible plant carbohydrates could facilitate disease prevention did not receive much attention from the nutritional community.

In the 1970s, several researchers including Burkitt (1972), Trowell (1972a), Painter (1973), and Walker (1974) proposed that the low prevalence of cardiovascular diseases in developing countries was largely due to the consumption of a diet rich in fiber. Following these initial observations, several early epidemiological investigations supported an inverse relationship between dietary fiber consumption and the incidence of coronary heart disease (Morris et al 1977; Yano et al 1978; Kromhout et al 1982).

One of the earliest researchers to provide experimental evidence in support of a protective role of dietary fiber against coronary heart disease was David Kritchevsky. Kritchevsky was intrigued that saturated fat feeding induced atherosclerosis in rabbits fed semi-purified diets, but not in chow-fed rabbits. Kritchevsky hypothesized that this discrepancy was likely due to the cardioprotective effects associated with dietary fiber components in the commercial chow diets (Kritchevsky 1964). The results from Kritchevsky's subsequent experiments supported his initial hypothesis of the cardioprotective effects of dietary fibers (Kritchevsky and Tepper 1965, 1968).

Although the dietary fiber hypothesis gained acceptance, a comprehensive definition of dietary fiber was needed in order to effectively study the epidemiological, physiological, and analytical aspects of these indigestible dietary components. However, the development of a comprehensive definition of dietary fiber that satisfied the distinct considerations of nutritionists, food technologists, and food chemists became the subject of great debate. In 1972, Trowell originally defined dietary fiber as 'the skeletal remains of plant cells that are resistant to hydrolysis of the enzymes of man' (Trowell 1972b). This definition was restricted to plant cell wall components including cellulose, hemicellulose, lignin, and other minor components such as waxes and cutin. This definition was subsequently amended to include plant storage polysaccharides such as gums and pectins, that have similar biological effects of traditional cell wall components, but did not have their origin in structures other than the cell wall (Trowell 1976).

With advances in analytical technology and a better understanding of the physiological implications of dietary fiber, the American Association of Cereal Chemists (2001) proposed the following comprehensive definition: 'dietary fiber is the edible parts of plants or analogous carbohydrates that are resistant to digestion and absorption in the human small intestine with complete or partial fermentation in the large intestine. Dietary fiber includes polysaccharides, oligosaccharides, lignin, and associated plant substances. Dietary fiber promotes beneficial physiological effects including laxation, and/or blood cholesterol attenuation, and/or blood glucose attenuation'. Ha and colleagues (2000) have criticized physiological-based fiber definitions that stress large intestinal fermentation without including possible interactions of dietary fiber with other dietary components in the upper gastrointestinal tract.

More recently, the Joint FAO/WHO Expert Consultation on Carbohydrates in Human Nutrition (Mann et al 2007) conceded that a re-evaluation of the fiber definition was warranted and proposed that dietary fiber should be defined as 'intrinsic plant cell wall polysaccharides'.

\section{Current understanding in fiber nutrition Classification of dietary fiber}

With years of research supporting the hypolipidemic effects and cardioprotective benefits associated with 
fiber consumption, recommendations for increased fiber consumption from health authorities around the world are justified (Heart and Stroke Foundation of Canada 2003; Lichtenstein et al 2006). However, it is also clear that actual fiber consumption in North America is much lower than what is currently recommended (James et al 2003). This discrepancy may be due to the confusion created by the broad range of substances that are classified under the umbrella of 'dietary fiber' and the lack of knowledge concerning the effect of different fibers on human health (Shamliyan et al 2006). Dietary fibers are typically classified according to various physical-chemical and physiological criteria including solubility, viscosity, and fermentability (James et al 2003). Generally, soluble fibers such as guar gum (GG), pectin, and psyllium are highly viscous and are readily fermented to short-chain fatty acids (SCFA) in the large bowel in comparison with insoluble fibers such as cellulose (James et al 2003). Furthermore, soluble fibers elicit a much more pronounced hypolipidemic and hypoglycemic response than their insoluble counterparts (Fernandez 2001).

\section{The variable hypocholesterolemic response to soluble fiber consumption}

Consumption of water-soluble, viscous-forming fibers such as GG, pectin, and psyllium has consistently been shown to reduce plasma cholesterol in human subjects (Brown et al 1999; Knopp et al 1999; Schneeman 1999; Butt et al 2007). The extent of cholesterol lowering in response to fiber consumption is variable, depending on a number of factors including the nature of the background diet, type of dietary fiber, length of adaptation period, and amount of fiber consumed. Furthermore, much of this variability in the plasma lipid-changes reported in response to dietary fiber consumption is thought to have a genetic basis (Cara et al 1992).

The greatest hypolipidemic effects in response to soluble fiber consumption are seen in animal model investigations where experimental conditions allow fiber intake to be above what is typically observed in humans. Fernandez and colleagues have reported a reduction in total plasma cholesterol up to $43 \%$ in guinea pigs fed a high cholesterol $(0.25 \%)$ diet supplemented with $12.5 \%$ GG (Shen et al 1998). Interestingly, the cholesterol-lowering effect of GG is more pronounced when consumed with a high-cholesterol diet compared with a cholesterol-free diet. In rats fed 7.5\% GG, Moundras and colleagues (1997) reported total plasma cholesterol reductions of 14 and $32 \%$ with cholesterolfree or cholesterol-enriched $(0.3 \%)$ diets, respectively. Similarly, guinea pigs fed diets supplemented with $12.5 \% \mathrm{GG}$ responded with a $22 \%$ reduction in plasma total cholesterol on a low-cholesterol (0.04\%) diet, but demonstrated a $38 \%$ reduction in total plasma cholesterol on a high-cholesterol $\operatorname{diet}(0.25 \%)$ (Fernandez 1995).

Clinical investigations typically report low-density lipoprotein (LDL)-cholesterol reductions in the range of $6 \%-15 \%$ (Fernandez 2001). Following the consumption of $14 \mathrm{~g} / \mathrm{d}$ of psyllium in twenty type 2 diabetic patients for 6 weeks, Sierra and colleagues (2002) observed a reduction in total and LDL-cholesterol by $7 \%$ and $9 \%$, respectively. In addition, a large reduction (25\%) in LDL-cholesterol was observed in 24 healthy volunteers receiving $9 \mathrm{~g}$ of GG per day for 4 weeks (Khan et al 1981). Alternatively, a meta analysis of 67 controlled studies by Brown and colleagues (1999) suggested that consumption of 2-10 g of major dietary fibers such as pectin, oat bran, guar gum and psyllium resulted in small but significant reductions in total and LDL-cholesterol. Finally, postprandial circulating lipid concentrations have also been shown to shift favorably following the consumption of dietary fiber. Consumption of a high fiber test meal with $15.7 \mathrm{~g}$ of beta-glucan reduced postprandial cholesterol concentrations below the fasting level in 11 healthy men in comparison to a low fiber test meal with $5.0 \mathrm{~g}$ of beta-glucan (Bourdon et al 1999).

\section{Cholesterol-lowering mechanisms of soluble fibers}

Our traditional understanding of how dietary soluble fibers alter cholesterol metabolism and reduce circulating cholesterol concentrations is illustrated in Figure 1. It is widely accepted that soluble fibers act primarily in the intestine to promote secondary responses in the liver and peripheral circulation from direct effects within the intestinal lumen (Fernandez et al 1997; Schneeman 1999). One of the primary actions of soluble fibers in the intestine is to reduce dietary fat and cholesterol uptake (Schneeman 1999). Reductions in apparent intestinal cholesterol absorption have been reported in both human subjects (Simons et al 1982) and animal models (Levrat-Verny et al 2000). Furthermore, in vitro cholesterol uptake into everted sacs of rat proximal small intestine from a solution of micelles was reduced by $40 \%$ when GG was included in the incubation medium (Gee et al 1983). It has been proposed that the viscosity associated with soluble fibers interferes with key physiological events in the cholesterol absorptive process. These interference mechanisms include direct binding of cholesterol within the intestinal lumen (Andersson 1992), interference with the diffusion of luminal cholesterol toward the epithelial cell surface 


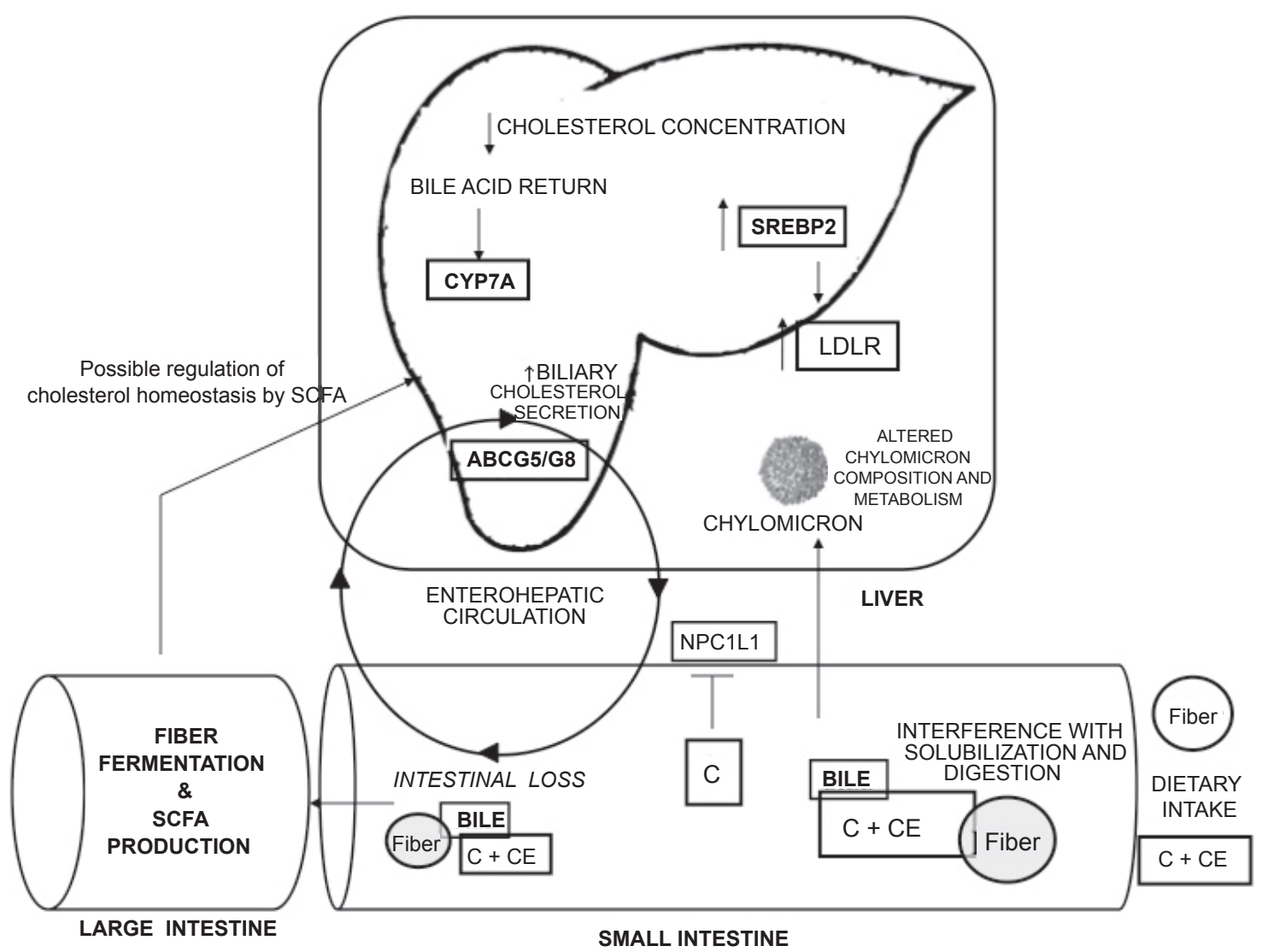

Figure I Potential mechanisms responsible for the hypocholesterolemic effects associated with the consumption of soluble fibers. Copyright $@ 2007$. Adapted with permission from Rideout TC, Yuan Z, Bakovic M, et al. 2007. Guar gum consumption increases hepatic nuclear SREBP2 and LDL receptor expression in pigs fed an atherogenic diet. J Nutr, 137:568-72.

Abbreviations: ABCG5/G8, ATP-binding cassette transporter G5 and G8; C, cholesterol; CE, cholesteryl ester; CYP7A, cholesterol 7a-hydroxylase; GG, guar gum; LDLr, low-density lipoprotein receptor; NPCILI, Niemann-Pick-C-I like I; SCFA, short-chain fatty acids; SREBP2, sterol regulatory element binding protein 2.

(Schneeman 1999), and/or antagonization of the cholesterol emulsification process (Minekus et al 2005). While our knowledge concerning the molecular mechanisms responsible for cholesterol balance within intestinal enterocytes has greatly advanced in recent years, the reduction in cholesterol absorption in response to soluble fiber consumption is most often attributed to viscosity with very little emphasis on the potential regulation of proteins that mediate intestinal cholesterol absorption (Dikeman and Fahey 2006).

The hypocholesterolemic effects of soluble fibers are also believed to result from an interference with the enterohepatic circulation of bile acids (Fernandez 2001). Marlett and colleagues (1994) concluded that the consumption of oat bran in normolipidemic young men decreased serum cholesterol by altering the composition of the enterohepatic bile acid pool and increasing the fecal loss of total bile acids. These observations were further demonstrated in human clinical studies by Lia and colleagues (1995), Andersson and colleagues (2002), and Ellegård and Andersson (2007). The dietary supplementation of psyllium at a level of $10 \%$ is associated with a dose-dependent increase in fecal bile acid excretion in rats (Buhman et al 2000). Similarly, native amylo-maize resistant starch, a modified starch with similar physiological effects to soluble fibers, has been shown to increase the fecal excretion of primary bile acids in human subjects (van Munster et al 1994). Interestingly, while GG was traditionally thought to reduce intestinal bile acid uptake, a series of reports in the late 1990's suggest that intestinal bile acid flux to the liver may actually be increased in response to GG consumption (Favier et al 1998; Moundras et al 1997; Moriceau et al 2000). Therefore, the traditional mechanisms by which GG reduces plasma cholesterol concentrations may have to be re-evaluated.

The reduction in intestinal cholesterol absorption and bile acid uptake in response to soluble fiber consumption are believed to alter hepatic cholesterol homeostasis by effectively reducing cholesterol concentrations in the liver by two related mechanisms. Firstly, a decrease in the delivery of dietary cholesterol to the liver through chylomicron remnants results in a direct reduction in the hepatic cholesterol pool (Fernandez 2001). The dietary supplementation of GG at 
$12.5 \%$ has been shown to reduce hepatic free and esterified cholesterol levels by $21 \%$ and $16 \%$, respectively, in guinea pigs (Fernandez 1995). Secondly, an increase in the fecal loss of bile acids and a reduction in the enterohepatic bile acid pool size may stimulate the liver to produce more bile acids from cholesterol, thus reducing hepatic free cholesterol concentrations (van Bennekum et al 2005).

The increased hepatic demand for cholesterol from the fiber-induced reduction in hepatic microsomal free cholesterol pool can be met by increasing the uptake of lipoprotein cholesterol from the plasma, the release of free cholesterol from intracellular storage of cholesteryl ester and membrane cholesterol, or by increasing hepatic cholesterol synthesis (Fernandez 2001; Rideout et al 2007). Consumption of various types of soluble fibers has been shown to increase the fractional catabolic rate of LDL (Vergara-Jimenez et al 1998) and hepatic LDLr expression (Fernandez 1995; Fukishima et al 2001; Han et al 2004).

A compensatory increase in the expression of hepatic HMG-CoA reductase, the rate-limiting enzyme in cholesterol biosynthesis, is thought to account for the reduction in the hepatic free cholesterol pool following the consumption of GG (Favier et al 1998), manufactured soluble fiber from wood chips (Chai et al 2003), psyllium (Buhman et al 2000), and pectin (Garcia-Diez et al 1996). It has been suggested that GG elicits a hypocholesterolemic effect even in the face of an increase in hepatic cholesterol synthesis, as the increase in HMG-CoAr activity is not sufficient to compensate for fecal steroid loss in rats fed a diet supplemented with 7.5\% GG (Moundras et al 1997).

The reduction in hepatic free cholesterol concentrations following soluble fiber consumption is thought to lead to a modification of hepatic lipoprotein metabolism. Soluble fiber consumption has been associated with a reduction in hepatic apolipoprotein B secretion and the formation of large triglyceride-rich, cholesteryl-ester-depleted very LDL (VLDL) particles (Fernandez 2001). This change in the endogenous cholesterol pathway results in a VLDL particle that is less prone to IDL and LDL conversion via extrahepatic lipases and LDL particles with a high peak density (Roy et al 2000; Fernandez 2001).

The production of SCFA by bacterial fermentation in the large intestine is thought to have multiple health benefits and may be involved in mediating the hypocholesterolemic effects of dietary fiber (Chen et al 1984). Furthermore, dietary fibers with unique physiochemical properties elicit differential SCFA profiles in the large intestine (Rideout et al 2008). Strong evidence for this SCFA-induced hypocholesterolemic effect from fiber fermentation comes from the observation that the cholesterol-lowering action of beet pulp disappears in rats following the removal of the cecum, both the cecum and colon, but not upon the removal of the colon alone (Nishimura et al 1993). However, the reduction in total cholesterol, LDL-cholesterol, and apolipoprotein B concentrations in ileostomy subjects fed an oat bran based high fiber diet suggests that large intestinal fiber fermentation is not necessary for cholesterol-reductions in human subjects (Zhang et al 1992). Particular emphasis has been placed on the role of butyrate as a signaling molecule that is important in regulating intestinal function and systemic cholesterol metabolism (Marcil et al 2002). Moreover, it has recently been suggested that butyrate may impair the assembly of triglyceride-rich lipoproteins within intestinal enterocytes, as a decrease in the basolateral efflux of cholesteryl ester in response to butyrate has been demonstrated in Caco- 2 cells (Marcil et al 2003).

\section{Research priorities in dietary fiber and cholesterol metabolism Individual physiological responses to dietary fibers}

The future of functional foods in modulating human health is closely connected to the concept of 'personalized nutrition' (Vakili and Caudill 2007). The ability to specifically tailor dietary treatment based on individual genetic polymorphisms will bolster the importance of the diet in disease prevention and potentially revolutionize the world's health care system. Although dietary factors are effective modulators of cellular metabolism at the molecular level, it is becoming increasingly clear that there is considerable variability in the response of plasma lipids to various nutritional lipid-lowering therapies including plant sterols (Jones et al 1999) and dietary fibers (Cara et al 1992). This variable inter-individual response is in part due to single base variations within gene sequences that function as molecular targets of dietary bioactive components (Trujillo et al 2006). Although the influence of genetic polymorphisms on the lipoprotein profile in response to plant sterols (Plat and Mensink 2002), dietary cholesterol (Robitaille et al 2007), and soy isoflavones (Hall et al 2006) have been extensively studied, the role of genotype in determining the hypocholesterolemic effects of dietary fibers has been the subject of few investigations.

Apolipoprotein E (ApoE), the major apoprotein component of chylomicrons, is polymorphic and thought to influence the response of plasma lipids to dietary intervention 
strategies (Zannis 1986). Jenkins' group has addressed the association between gene polymorphisms and the lipidlowering response to dietary fiber consumption in three separate publications. In the first of these publications, Jenkins and colleagues (1993) examined the association between the three common apo E alleles (E2, E3, and E4) and the plasma cholesterol response observed in 67 volunteers who completed a controlled dietary intervention that included a 2 week consumption of oat bran or wheat bran at a level of $6.8 \mathrm{~g}$ fiber/1000 kcal. Total cholesterol, LDL-cholesterol, and apolipoprotein B (ApoB) concentrations were significantly lower in subjects heterozygous for the E2 allele following fiber consumption in comparison to the E3 homozygotes and E4 carriers. However, a more recent study by $\mathrm{Wu}$ and colleagues (2007), using 22,915 free-living participants from the European Prospective Investigation of Cancer Norfolk study, suggest that ApoE genotype does not reflect changes in plasma cholesterol profile in response to dietary fat or fiber intake. The authors propose that the apparent controversy surrounding the role of ApoE phenotypes in the serum lipid response to diet is likely due to the lack of statistical power associated with the small sample size used as part of the experimental design in previous investigations. In a second study from the Jenkins lab, Hegele and colleagues (1993) hypothesized that polymorphisms in genes regulating ApoB metabolism, including the low-density lipoprotein receptor (LDLr), ApoB, apolipoprotein CIII (ApoCIII), and hepatic lipase, could influence the fiber-related reduction of ApoB containing lipoproteins. The results of this investigation suggested that LDLr genotype had a significant impact on the plasma lipid response following dietary fiber consumption. Finally, a preliminary investigation by Hegele and colleagues (1997) suggested that genetic variations in the fatty acid binding protein 2 gene, involved in regulating intestinal fatty acid absorption and intracellular transport (Hanhoff et al 2002), may be involved in modulating the reductions in plasma cholesterol in response to dietary fiber consumption. However, the authors suggest that the results be interpreted with caution due to the small sample size $(n=43)$ and the marginality associated with some of the statistical outcomes.

As can be seen from the above discussion, published reports on the role of gene polymorphism in the cholesterol-lowering response to soluble fibers are few and often limited by small sample size. The hypocholesterolemic effects of dietary fibers are initiated by primary intestinal events related to the digestion and absorption of dietary fat and cholesterol (Lairon 1996). Therefore, investigation into the association between polymorphisms in genes that regulate intestinal triglyceride and cholesterol digestion and plasma lipoprotein outcomes in response to soluble fiber consumption may be prudent. Indeed, pancreatic triglyceride lipase and carboxyl ester lipase, two enzymes involved in intestinal triglyceride and cholesterol digestion, have been shown to be highly polymorphic and may therefore explain some of the variability associated with dietary fiber consumption (Lidberge et al 1992; Cao and Hegele 2003).

\section{The 'omics' approach to dietary fiber research}

The focus of modern day nutrition research is shifting with the advent of novel, high-throughput investigative tools that permit an examination of how nutrients affect the global expression and characterization of transcripts, proteins, and metabolites (Afman and Muller 2006). The application of multiplexing technology involved in nutrigenomics, nutriepigenetics, transcriptomics, proteomics, and metabolomics will advance our understanding of nutrient-gene-metabolite interactions and ultimately lead to the identification of novel biomarkers of disease risk and the development of effective functional food products (Trujillo et al 2006). Although this 'omics' approach to nutrition may currently be limited by its high expense and the need for highly qualified individuals to analyze and integrate the vast amount of data that are generated, it has given us valuable insight into how the consumption of various dietary ingredients, including dietary fat (Kim et al 2004), soy isoflavones (Fuchs et al 2007b), dietary zinc (Blanchard et al 2001), and plant sterols (Calpe-Berdiel et al 2007), affect global gene expression and cell metabolism. However, very few published reports have applied 'omic' technology to the dietary fiber field. One exception has been a recently published report by Fuchs and colleagues (2007a) who applied a proteomic approach to study the effect of flaxseed consumption on differentially expressed genes in human peripheral blood mononuclear cells. Flaxseed consumption at a level of $0.4 \mathrm{~g} / \mathrm{kg} \mathrm{BW}$ for 7 days, resulted in 16 differentially expressed proteins compared with the control group. This differential protein expression pattern included enhanced levels of peroxiredoxin and reduced expression of long-chain fatty acid $\beta$-oxidation multienzyme complex and glycoprotein IIIa/II, suggestive of an antithrombotic and antiinflammatory effect from the flaxseed treatment. Microarray technology has also been employed to study the mechanisms by which intestinal SCFA produced from fiber fermentation in the large intestine affect the progression of colon cancers (Mariadason et al 2000; Daly et al 2006). Given the heterogeneity associated with dietary fibers, it is likely that fiber 
sources with different physiochemical properties will produce a unique metabolic 'footprint'. Furthermore, the movement of nutrition research from a reductionist science to a systems biology approach will allow the integration of complex biological information at the gene, protein, and metabolite level and advance our understanding of the role of dietary soluble fiber in achieving optimal personalized health.

\section{Regulation of genes and protein expression within the enterohepatic loop}

In order to avoid the potential atherogenic consequences associated with elevated plasma cholesterol, elaborate regulatory mechanisms have evolved to balance the intestinal absorption and de novo synthesis of cholesterol (the major input pathways) with biliary cholesterol and bile acid excretion (the major output pathways) (Lammert and Wang 2005). Our understanding of the molecular regulators of enterohepatic cholesterol and bile acid metabolism has been greatly advanced through the characterization of the major proteins involved in maintaining sterol balance at the intestinal and hepatic levels (Figure 2). Within the intestine, cholesterol absorption and efflux appear to be regulated largely through the brush-border membrane proteins Niemann-Pick $\mathrm{C}$ like 1 and ATP-binding cassette G5 and G8, respectively (Kruit et al 2006). At the hepatic level, the clearance of plasma LDL and HDL-cholesterol occurs through the LDLr and the scavenger receptor class B, type 1 (SR-B1) while cholesterol synthesis and oxidation is precisely controlled through activity of the rate-limiting enzymes HMG-CoAr and cholesterol 7 $\alpha$-hydroxylase (CYP7A1), respectively (Dietschy et al 1993). Furthermore, the influence of hepatic ABCG5/G8 expression on biliary cholesterol secretion and intestinal cholesterol absorption has only recently been recognized (Wu et al 2004; Rideout and Fan 2008). Finally, numerous nuclear receptors (eg, LXR, FXR) and transcription factors (eg, SREBP2) have been shown to be critical in modulating transcriptional events and maintaining cholesterol homeostasis within the enterohepatic loop (Wong et al 2006).

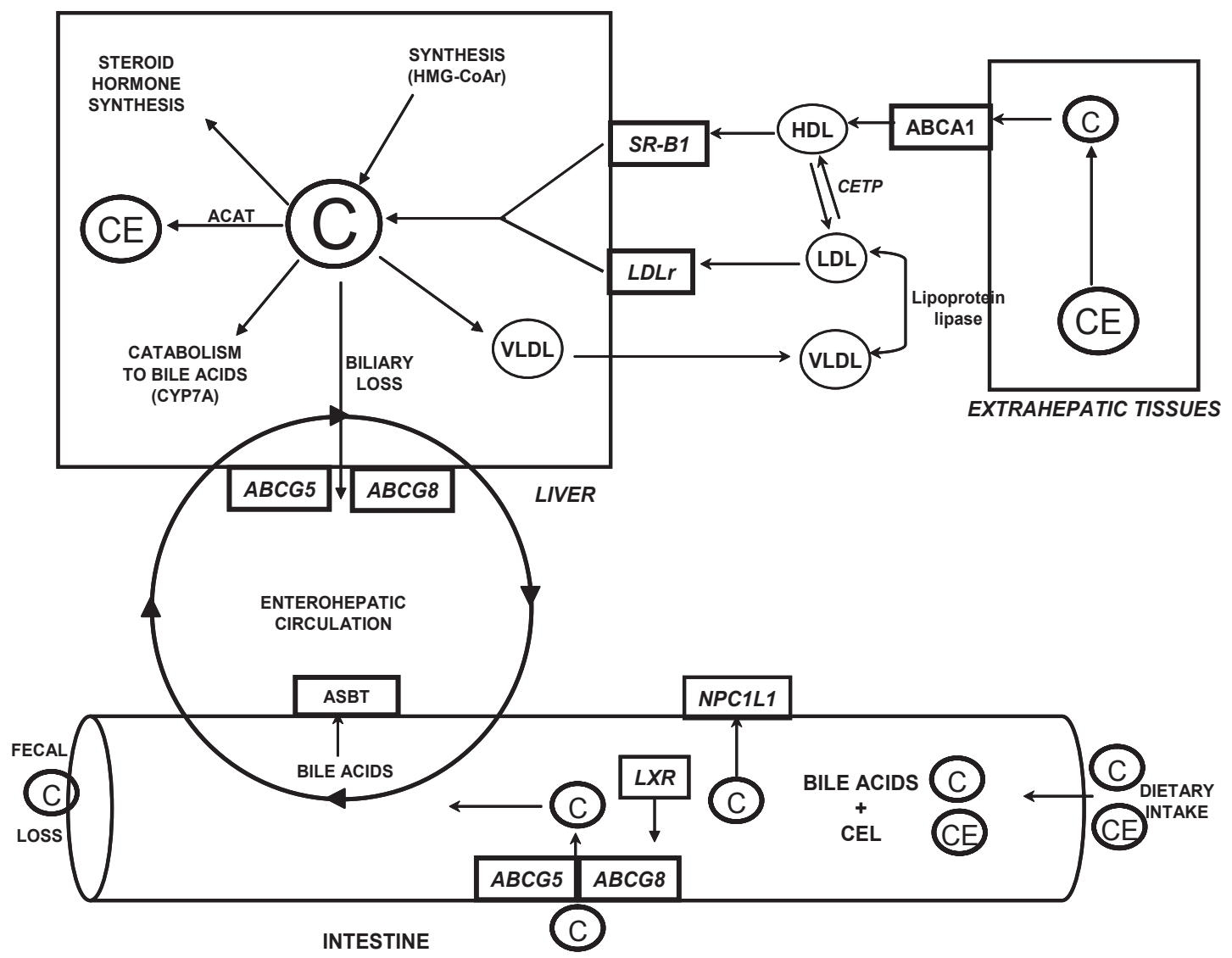

Figure 2 Major pathways involved in regulating the enterohepatic circulation of cholesterol. Copyright @) 2007. Adapted with permission from Rideout TC, Yuan Z, Bakovic M, et al. 2007. Guar gum consumption increases hepatic nuclear SREBP2 and LDL receptor expression in pigs fed an atherogenic diet. J Nutr, 137:568-72.

Abbreviations: ABCG5,ATP-binding cassette transporter G5;ABCG8,ATP-binding cassette transporter G5;ACAT, acyl-coenzyme A:cholesterol acyltransferase;ASBT, apical sodium-dependent bile acid transporter; C, cholesterol; CE, cholesteryl ester; CEL, carboxyl ester lipase; CYP7A, cholesterol 7 $\alpha$-hydroxylase; HDL, high-density lipoprotein; ABCAI, ATP-binding cassette transporter AI; HMG-CoAr, 3-hydroxy-3-methylglutaryl-CoA reductase; LDLr, low-density lipoprotein receptor; NPCILI, Niemann-Pick-C-I like I; SR-BI, scavenger receptor class B, type I;VLDL, very-low density lipoprotein. 
Information concerning the effect of dietary fiber consumption on the expression of genes that regulate intestinal cholesterol absorption and hepatic sterol balance is limited. For the most part, published reports on the molecular mechanisms associated with the hypocholesterolemic effects of dietary fibers have pertained to the hepatic mRNA expression of HMG-CoAr and CYP7A1 (Horton et al 1994; Kishida et al 2002; Han et al 2004). Although these studies have provided insight into how fibers may affect metabolism at the gene level, the expression of a gene from DNA to protein is a complex process with control mechanisms that regulate multiple steps including transcription rate, nuclear export and mRNA localization, transcript stability, translational efficiency, protein degradation, and post-translational processing (Pradet-Balade et al 2001; Hanash and Beretta 2002; Yang et al 2008). Therefore, cellular mRNA levels are often unreliable indicators of protein abundances, and the abundance of any particular protein does not necessarily reflect its catalytic activity (Hanash and Beretta 2002). In an effort to more fully characterize the hypocholesterolemic effects of soluble fiber at the molecular level, our recent work proposes several novel mechanisms by which GG may exert its effects. Firstly, our data suggests that the hypocholesterolemic effects of GG consumption are mediated by a reduction in hepatic free-cholesterol concentration and an associated SREBP2-dependent increase in hepatic LDLr mRNA and protein expression (Rideout et al 2007). At the transcriptional level, hepatic cholesterol metabolism is tightly controlled through SREBP2, a nuclear receptor that binds to sterol response elements in the promoter region of a multitude of target genes (Hua et al 1993). In the absence of a change in SREBP2 mRNA, we observed an increase in the cytoplasmic precursor and nuclear active forms of SREBP2 without a corresponding increase in the cytoplasmic mature form of the protein in response to GG consumption. This differential expression pattern suggests GG consumption may have increased nuclear SREBP2 expression through mechanisms independent of protease-dependent cleavage at the Golgi.

Secondly, in response to GG consumption we observed an increase in hepatic ABCG5/G8 mRNA and protein expression and biliary cholesterol concentration in comparison to the control-fed pigs (Rideout 2007; Rideout and Fan 2008). Traditionally, by interfering with the enterohepatic circulation and intestinal uptake of bile acids, soluble fiber consumption is believed to modulate whole-body cholesterol excretion through stimulation of cholesterol catabolism to bile acids (Trautwein et al 1998). However, an additional quantitatively important route of cholesterol excretion occurs through the biliary secretion and eventual fecal loss of free cholesterol (Spritz et al 1965). Our recent data suggest that the stimulation of biliary free cholesterol secretion through hepatic ABCG5/G8 may exist as a novel mechanism by which GG consumption stimulates whole-body cholesterol loss and effectively reduces plasma cholesterol concentrations (Rideout 2007; Rideout and Fan 2008).

Both targeted traditional molecular approaches and novel high-throughput technology may be employed to gain a more comprehensive understanding of how dietary soluble fiber consumption regulates gene and protein express patterns. Unlike other dietary bioactive components that are thought to directly regulate the transcription of hepatic cholesterol-responsive genes (Mezei et al 2003), dietary fiber components are not absorbed from the gastrointestinal tract and therefore are generally thought to influence hepatic cholesterol homeostasis through secondary signaling systems and metabolites (Fernandez 1995). Therefore, to fully delineate the molecular events by which soluble fiber consumption modulates peripheral cholesterol metabolism, it will be critical to determine the molecular signals that are involved in mediating these secondary effects.

\section{Conclusions}

Although dietary fiber may be considered a 'dinosaur' among other functional foods, it should not be perceived as extinct. The future relevance of functional fiber-based foods will depend on the ability to integrate consumer health concerns with a comprehensive understanding of the health benefits and diseaseprevention potential of unique varieties of soluble fibers. Due to the specific physical-chemical attributes that are associated with fibers that originate from different plant sources and produced with alternative processing technologies, dietary fibers are unique functional ingredients that have wide-ranging appeal in the functional food community. While the hypocholesterolemic effects associated with soluble fiber consumption are clear, a comprehensive understanding of these effects, particularly at the molecular level, will require additional exploration. Advances in understanding the variable interindividual response to dietary soluble fibers and the effect of fiber consumption on expression patterns at the gene, protein and metabolite level will advance through application of traditional molecular tools and novel highthroughput technology. Detailed knowledge of the mechanisms by which different soluble fibers reduce plasma cholesterol concentrations is paramount to developing novel fiber-based "cocktails" and portfolio-type diets that target specific metabolic pathways to gain maximal cholesterol reductions. 


\section{Disclosure}

The authors report no conflicts of interest in this work.

\section{References}

Adams F. (ed). 1939. The genuine works of Hippocrates. The Williams and Wilkins Company, Baltimore, MD, USA.

Afman L, Muller M. 2006. Nutrigenomics: from molecular nutrition to prevention of disease. J Am Diet Assoc, 106:569-76.

American Association of Cereal Chemists. 2001. The definition of dietary fiber. Cereal Foods World, 46:112-26.

Anderson JW, Allgood LD, Lawrence A, et al. 2000a. Cholesterol-lowering effects of psyllium intake adjunctive to diet therapy in men and women with hypercholesterolemia: meta-analysis of 8 controlled trials. Am J Clin Nutr, 71:472-9.

Anderson JW, Davidson MH, Blonde L, et al. 2000b. Long-term cholesterol-lowering effects of psyllium as an adjunct to diet therapy in the treatment of hypercholesterolemia. Am J Clin Nutr, 71:1433-8.

Anderson JW, Major AW. 2002. Pulses and lipaemia, short- and long-term effect: potential in the prevention of cardiovascular disease. Br J Nutr, 88(Suppl 3):S263-71.

Anderson JW, Story L, Sieling B, et al. 1984. Hypocholesterolemic effects of oat-bran or bean intake for hypercholesterolemic men. Am J Clin Nutr, 40:1146-55.

Anderson JW, Zeigler JA, Deakins DA, et al. 1991. Metabolic effects of high-carbohydrate, high-fiber diets for insulin-dependent diabetic individuals. Am J Clin Nutr, 54:936-43.

Andersson H. 1992. The ileostomy model for the study of carbohydrate digestion and carbohydrate effects on sterol excretion in man. Eur $J$ Clin Nutr, 46:S69-76.

Andersson M, Ellegård L, Andersson H. 2002. Oat bran stimulates bile acid synthesis within $8 \mathrm{~h}$ as measured by 7alpha-hydroxy-4-cholesten-3-one. Am J Clin Nutr, 76:1111-6

Atwater WO. 1900. Discussion of the terms digestibility, availability, and fuel value. 12th Annual report. Storrs. Agricultural Experimental Station. Storrs, Connecticut. p. 69.

Bell LP, Hectorne K, Reynolds H, et al. 1989. Cholesterol-lowering effects of psyllium hydrophilic mucilloid. Adjunct therapy to a prudent diet for patients with mild to moderate hypercholesterolemia. JAMA, 261:3419-23.

Blanchard RK, Moore JB, Green CL, et al. 2001. Modulation of intestinal gene expression by dietary zinc status: effectiveness of cDNA arrays for expression profiling of a single nutrient deficiency. Proc Natl Acad Sci US A, 98:13507-13.

Bourdon I, Yokoyama W, Davis P, et al. 1999. Postprandial lipid, glucose, insulin, and cholecystokinin responses in men fed barley pasta enriched with beta-glucan. Am J Clin Nutr, 69:55-63.

Braaten JT, Wood PJ, Scott FW, et al. 1994. Oat beta-glucan reduces blood cholesterol concentration in hypercholesterolemic subjects. Eur J Clin Nutr, 48:465-74.

Brown L, Rosner B, Willett WW, et al. 1999. Cholesterol-lowering effects of dietary fiber: a meta-analysis. Am J Clin Nutr, 69:30-42.

Buhman KK, Furumoto EJ, Donkin SS, et al. 2000. Dietary psyllium increases expression of ileal apical sodium-dependent bile acid transporter mRNA coordinately with dose-responsive changes in bile acid metabolism in rats. $J$ Nutr, 130:2137-42.

Burkitt DP, Walker AR, Painter NS. 1972. Effect of dietary fiber on stools and the transit-times, and its role in the causation of disease. Lancet, 2:1408-12.

Butt MS, Shahzadi N, Sharif MK, et al. 2007. Guar gum: a miracle therapy for hypercholesterolemia, hyperglycemia and obesity. Crit Rev Food Sci Nutr, 47:389-96.

Calpe-Berdiel L, Escola-Gil JC, Julve J, et al. 2007. Differential intestinal mucosal protein expression in hypercholesterolemic mice fed a phytosterol-enriched diet. Proteomics, 7:2659-66.

Cao H, Hegele RA. 2003. DNA polymorphisms of lipase related genes. J Hum Genet, 48:443-6.
Cara L, Dubois C, Borel P, et al. 1992. Effects of oat bran, rice bran, wheat fiber, and wheat germ on postprandial lipemia in healthy adults. $A m J$ Clin Nutr, 55:81-8.

Castro IA, Barroso LP, Sinnecker P. 2005. Functional foods for coronary heart disease risk reduction: a meta-analysis using a multivariate approach. Am J Clin Nutr, 82:32-40.

Chai YM, Lim BK, Lee JY, et al. 2003. Effects of manufactured soluble dietary fiber from Quercus mongolica on hepatic HMG-CoA reductase and lipoprotein lipase activities in epididymal adipose tissue of rats fed high cholesterol diets. J Med Food, 6:329-36.

Chen WJ, Anderson JW, Jennings D. 1984. Propionate may mediate the hypocholesterolemic effects of certain soluble plant fibers in cholesterol-fed rats. Proc Soc Exp Biol Med, 175:215-8.

Daly K, Shirazi-Beechey SP. 2006. Microarray analysis of butyrate regulated genes in colonic epithelial cells. DNA Cell Biol, 25:49-62.

Dietschy JM, Turley SD, Spady DK. 1993. Role of liver in the maintenance of cholesterol and low density lipoprotein homeostasis in different animal species, including humans. J Lipid Res, 34:1637-59.

Dikeman CL, Fahey GC. 2006. Viscosity as related to dietary fiber: a review. Crit Rev Food Sci Nutr, 46:649-63.

Ellegård L, Andersson M. 2007. Oat bran rapidly increases bile acid excretion and bile acid synthesis: an ileostomy study. Eur J Clin Nutr, 61: 938-45.

Favier ML, Bost PE, Demigne C, et al. 1998. The cholesterol-lowering effect of guar gum in rats is not accompanied by an interruption of bile acid cycling. Lipids, 33:765-71.

Fernandez ML. 1995. Distinct mechanisms of plasma LDL lowering by dietary fiber in the guinea pig: specific effects of pectin, guar gum, and psyllium. J Lipid Res, 36:2394-404.

Fernandez ML. 2001. Soluble fiber and indigestible carbohydrate effects on plasma lipids and cardiovascular risks. Curr Opin in Lipidol, 12:35-40.

Fernandez ML, Vergara-Jimenez M, Conde K, et al. 1997. Regulation of apolipoprotein B-containing lipoproteins by dietary soluble fiber in guinea pigs. Am J Clin Nutr, 65:814-22.

Fernandez ML, Wilson TA, Conde K, et al. 1999. Hamsters and guinea pigs differ in their plasma lipoprotein cholesterol distribution when fed diets varying in animal protein, soluble fiber, or cholesterol content. J Nutr, 129:1323-32.

Fuchs D, Piller R, Linseisen J, et al. 2007a. The human peripheral blood mononuclear cell proteome responds to a dietary flaxseed-intervention and proteins identified suggest a protective effect in atherosclerosis. Proteomics, 7:3278-88.

Fuchs D, Vafeiadou K, Hall WL, et al. 2007b. Proteomic biomarkers of peripheral blood mononuclear cells obtained from postmenopausal women undergoing an intervention with soy isoflavones. Am J Clin Nutr, 86:1369-75.

Fukushima M, Ohashi T, Fujiwara Y, et al. 2001.Cholesterol-lowering effects of maitake (Grifola frondosa) fiber, shiitake (Lentinus edodes) fiber, and enokitake (Flammulina velutipes) fiber in rats. Exp Biol Med, 226:758-65.

Garcia-Diez F, Garcia-Mediavilla V, Bayon JE, et al. 1996. Pectin feeding influences fecal bile acid excretion, hepatic bile acid and cholesterol synthesis and serum cholesterol in rats. $J$ Nutr, 126:1766-71.

Gee JM, Blackburn NA, Johnson IT.1983. The influence of guar gum on intestinal cholesterol transport in the rat. Br J Nutr, 50:215-24.

Ha MA, Jarvis MC, Mann JI. 2000. A definition for dietary fiber. Eur J Clin Nutr, 54:861-4.

Hall WL, Vafeiadou K, Hallund J, et al. 2006. Soy-isoflavone-enriched foods and markers of lipid and glucose metabolism in postmenopausal women: interactions with genotype and equol production. Am J Clin Nutr, 83:592-600.

Han KH, Sekikawa M, Shimada K, et al. 2004. Resistant starch fraction prepared from kintoki bean affects gene expression of genes associated with cholesterol metabolism in rats. Exp Biol Med, 229:787-92. 
Hanash SM, Beretta LM. 2002. Operomics: integrated genomic and proteomic profiling of cells and tissues. Brief Funct Genomic Proteomic, 1:10-22.

Hanhoff T, Lucke C, Spener F. 2002. Insights into binding of fatty acids by fatty acid binding proteins. Mol Cell Biochem, 239:45-54.

Heart and Stroke Foundation of Canada. 2003. The growing burden of heart disease and stroke in Canada. Ottawa, Canada.

Hegele RA, Wolever TM, Story JA, et al. 1997. Intestinal fatty acid-binding protein variation associated with variation in the response of plasma lipoproteins to dietary fibre. Eur J Clin Invest, 27:857-62.

Hegele RA, Zahariadis G, Jenkins AL, et al. 1993. Genetic variation associated with differences in the response of plasma apolipoprotein B levels to dietary fibre. Clin Sci, 85:269-75.

Horton JD, Cuthbert JA, Spady DK. 1994. Regulation of hepatic 7 alpha-hydroxylase expression by dietary psyllium in the hamster. J Clin Invest, 93:2084-92.

Hua X, Yokoyama C, Wu J, et al. 1993. SREBP-2, a second basic-helix-loop-helix-leucine zipper protein that stimulates transcription by binding to a sterol regulatory element. Proc Natl Acad Sci U S A, 90:11603-7.

James SL, Muir JG, Curtis SL, et al. 2003. Dietary fiber: a roughage guide. Intern Med J, 33:291-6.

Jenkins DJ, Hegele RA, Jenkins AL, et al. 1993. The apolipoprotein E gene and the serum low-density lipoprotein cholesterol response to dietary fiber. Metabolism, 42:585-93.

Jones PJ, Ntanios FY, Raeini-Sarjaz M, et al. 1999. Cholesterol-lowering efficacy of a sitostanol-containing phytosterol mixture with a prudent diet in hyperlipidemic men. Am J Clin Nutr, 69:1144-50.

Khan AR, Khan GY, Mitchel A, et al. 1981. Effect of guar gum on blood lipids. Am J Clin Nutr, 34:2446-9.

Kim S, Sohn I, Ahn JI, et al. 2004. Hepatic gene expression profiles in a long-term high-fat diet-induced obesity mouse model. Gene, 340:99-109.

Kishida T, Nogami H, Ogawa H, et al. 2002. The hypocholesterolemic effect of high amylose cornstarch in rats is mediated by an enlarged bile acid pool and increased fecal bile acid excretion, not by cecal fermented products. J Nutr, 132:2519-24.

Knopp RH, Superko HR, Davidson M et al. 1999. Long-term blood cholesterol-lowering effects of a dietary fiber supplement. Am J Prev Med, 17:18-23

Kritchevsky DJ. 1964. Experimental atherosclerosis in rabbits fed cholesterol-free diets. Atheroscler Res, 4:103-5.

Kritchevsky DJ, Tepper SA. 1965. Factors affecting atherosclerosis in rabbits fed cholesterol-free diets. Life Sci, 4:1467-71

Kritchevsky DJ, Tepper SA. 1968. Experimental atherosclerosis in rabbits fed cholesterol-free diets: Influence of chow components. $J$, Atheroscler, Res, 8:357-69.

Kromhout D, Bosschieter EB, de Lezenne Coulander C. 1982. Dietary fiber and 10-year mortality from coronary heart disease, cancer, and all causes. The Zutphen study. Lancet, 2:518-22.

Kruit JK, Groen AK, van Berkel TJ, et al. 2006. Emerging roles of the intestine in control of cholesterol metabolism. World J Gastroenterol, 12:6429-9.

Lairon D. 1996. Dietary fibres: effects on lipid metabolism and mechanisms of action. Eur J Clin Nutr, 50:125-33.

Lairon D, Arnault N, Bertrais S, et al. 2005. Dietary fiber intake and risk factors for cardiovascular disease in French adults. Am J Clin Nutr, 82:1185-94

Lammert F, Wang DQ. 2005. New insights into the genetic regulation of intestinal cholesterol absorption. Gastroenterology, 129:718-34.

Levrat-Verny MA, Behr S, Mustad V, et al. 2000. Low levels of viscous hydrocolloids lower plasma cholesterol in rats primarily by impairing cholesterol absorption. J Nutr, 130:243-8.

Lia A, Hallmans G, Sandberg AS et al. 1995. Oat beta-glucan increases bile acid excretion and a fiber-rich barley fraction increases cholesterol excretion in ileostomy subjects. Am J Clin Nutr, 62:1245-51.
Lichtenstein AH, Appel LJ, Brands M, et al. 2006. Diet and lifestyle recommendations revision: a scientific statement from the American Heart Association Nutrition Committee. Circulation, 114:82-96.

Lidberg U, Nilsson J, Stromberg K, et al. 1992. Genomic organization, sequence analysis, and chromosomal localization of the human carboxyl ester lipase (CEL) gene and a CEL-like (CELL) gene. Genomics, 13:630-40.

Mann J, Cummings JH, Englyst HN, et al. 2007. AO/WHO Scientific Update on carbohydrates in human nutrition: conclusions. Eur J Clin Nutr, 61: S132-S137.

Marcil V, Delvin E, Garofalo C, et al. 2003. Levy E. Butyrate impairs lipid transport by inhibiting microsomal triglyceride transfer protein in Caco-2 cells. J Nutr, 133:2180-3.

Marcil V, Delvin E, Seidman E, et al. 2002. Modulation of lipid synthesis, apolipoprotein biogenesis, and lipoprotein assembly by butyrate. $\mathrm{Am}$ J Physiol Gastrointest Liver Physiol, 283:G340-6.

Marlett JA, Hosig KB, Vollendorf NW et al. 1994. Mechanism of serum cholesterol reduction by oat bran. Hepatology, 20:1450-7.

Mariadason JM, Corner GA, Augenlicht LH. 2000. Genetic reprogramming in pathways of colonic cell maturation induced by short chain fatty acids: comparison with trichostatin A, sulindac, and curcumin and implications for chemoprevention of colon cancer. Cancer Res, 60:4561-72.

McCance RA, Widdowson EM, Shackleton LRB. 1936. The nutritive value of fruits, vegetables, and nuts. Med Res Coun Spec Rep Serv no 213. HMSO, London.

Mezei O, Banz WJ, Steger RW, et al. 2003. Soy isoflavones exert antidiabetic and hypolipidemic effects through the PPAR pathways in obese Zucker rats and murine RAW 264.7 cells. J Nutr, 133:1238-43.

Minekus M, Jelier M, Xiao JZ, et al. 2005. Effect of partially hydrolyzed guar gum (PHGG) on the bioaccessibility of fat and cholesterol. Biosci Biotechnol Biochem, 69:932-8.

Moriceau S, Besson C, Levrat MA, et al. 2000. Cholesterol-lowering effects of guar gum: changes in bile acid pools and intestinal reabsorption. Lipids, 35:437-44.

Morris JN, Marr JW, Clayton DG. 1977. Diet and heart: a postscript. Br Med J, 2:1307-14.

Moundras C, Behr SR, Remesy C, et al. 1997. Fecal losses of sterols and bile acids induced by feeding rats guar gum are due to greater pool size and liver bile acid secretion. $J$ Nutr, 127:1068-76.

Naumann E, van Rees AB, Onning G, et al. 2006. Beta-glucan incorporated into a fruit drink effectively lowers serum LDL-cholesterol concentrations. Am J Clin Nutr, 83:601-5.

Nishimura N, Nishikawa H, Kiriyama S. 1993. Ileorectostomy or cecectomy but not colectomy abolishes the plasma cholesterol-lowering effect of dietary beet fiber in rats. $J$ Nutr, 123:1260-9.

Painter NS. 1973. A disease of Western civilization caused by a deficiency of dietary fiber. Trans Med Soc Lond, 89:85-91.

Plat J, Mensink RP. 2002. Relationship of genetic variation in genes encoding apolipoprotein A-IV, scavenger receptor BI, HMG-CoA reductase, CETP and apolipoprotein E with cholesterol metabolism and the response to plant stanol ester consumption. Eur J Clin Invest, $32: 242-50$.

Pradet-Balade B, Boulme F, Beug H, et al. 2001. Translation control: bridging the gap between genomics and proteomics? Trends Biochem Sci, 26:225-9.

Queenan KM, Stewart ML, Smith KN, et al. 2007. Concentrated oat betaglucan, a fermentable fiber, lowers serum cholesterol in hypercholesterolemic adults in a randomized controlled trial. Nutr J, 26:6.

Rideout TC. 2007. Guar Gum Consumption in Regulation of Enterohepatic Cholesterol Metabolism in Pigs Fed a High Fat Diet. PhD. Dissertation, University of Guelph, Guelph, ON, Canada.

Rideout TC, Liu Q, Wood P, et al. 2008. Nutrient utilisation and intestinal fermentation are differentially affected by the consumption of resistant starch varieties and conventional fibres in pigs. Br J Nutr, 99:984-92.

Rideout TC, Yuan Z, Bakovic M, et al. 2007. Guar gum consumption increases hepatic nuclear SREBP2 and LDL receptor expression in pigs fed an atherogenic diet. $J$ Nutr, 137:568-72. 
Rideout, TC, Fan MZ. 2008. Guar gum consumption enhances hepatic ABCG5/G8 expression and increases ileal cholesterol excretion in pigs [abstract]. FASEB J, 22:1092-13.

Ripsin CM, Keenan JM, Jacobs DR Jr, et al. 1992. Oat products and lipid lowering. A meta-analysis. JAMA, 267:3317-25.

Robitaille J, Houde A, Lemieux S, et al. 2007. The lipoprotein/lipid profile is modulated by a gene-diet interaction effect between polymorphisms in the liver $\mathrm{X}$ receptor-alpha and dietary cholesterol intake in French-Canadians. Br J Nutr, 97:11-8.

Roy S, Vega-Lopez S, Fernandez ML. 2000. Gender and hormonal status affect the hypolipidemic mechanisms of dietary soluble fiber in guinea pigs. J Nutr, 130:600-7.

Schneeman BO. 1999. Fiber, inulin and oligofructose: similarities and differences. J Nutr, 129:1424S-7S.

Shamliyan TA, Jacobs DR Jr, Raatz SK, et al. 2006. Are your patients with risk of CVD getting the viscous soluble fiber they need? J Fam Pract, 55:761-9.

Shen H, He L, Price RL, et al. 1998. Dietary soluble fiber lowers plasma LDL cholesterol concentrations by altering lipoprotein metabolism in female guinea pigs. J Nutr, 128:1434-41.

Sierra M, Garcia JJ, Fernandez N, et al. 2002. Therapeutic effects of psyllium in type 2 diabetic patients. Eur J Clin Nutr, 56:830-42.

Simons LA, Gayst S, Balasubramaniam S, et al. 1982. Long-term treatment of hypercholesterolaemia with a new palatable formulation of guar gum. Atherosclerosis, 45:101-8.

Spritz N, Ahrens EH Jr, Grundy S. 1965. Sterol balance in man as plasma cholesterol concentrations are altered by exchanges of dietary fats. J Clin Invest, 44:1482-93.

Steinberg D. 2004. An interpretive history of the cholesterol controversy: part 1. J Lipid Res, 45:1583-93.

Steinberg D. 2005. An interpretive history of the cholesterol controversy: part 2: The early evidence linking hypercholesterolemia to coronary disease in humans. J Lipid Res, 46:179-90.

Trautwein EA, Rieckhoff D, Erbersdobler HF. 1998. Dietary inulin lowers plasma cholesterol and triacylglycerol and alters biliary bile acid profile in hamsters. J Nutr, 128:1937-43.

Trowell H. 1972a. Dietary fiber and coronary heart disease. Rev Eur Etud Clin Biol, 17:345-9.

Trowell H. 1972b. Ischemic heart disease and dietary fiber. Am J Clin Nutr, 25:926-32.

Trowell H, Southgate DA, Wolever TM, et al. 1976. Letter: Dietary fiber redefined. Lancet, 1(7966):967.

Trujillo E, Davis C, Milner J. 2006. Nutrigenomics, proteomics, metabolomics, and the practice of dietetics. J Am Diet Assoc, 106:403-13.
Vakili S, Caudill MA. 2007. Personalized nutrition: nutritional genomics as a potential tool for targeted medical nutrition therapy. Nutr Rev, 65:301-15.

van Bennekum AM, Nguyen DV, Schulthess G, et al. 2005. Mechanisms of cholesterol-lowering effects of dietary insoluble fibres: relationships with intestinal and hepatic cholesterol parameters. Br J Nutr, 94:331-7.

van Munster IP, Tangerman A, Nagengast FM. 1994. Effect of resistant starch on colonic fermentation, bile acid metabolism, and mucosal proliferation. Dig Dis Sci, 39:834-42.

Venkatesan N, Devaraj SN, Devaraj H. 2007. A fibre cocktail of fenugreek, guar gum and wheat bran reduces oxidative modification of LDL induced by an atherogenic diet in rats. Mol Cell Biochem, 294:145-53.

Vergara-Jimenez M, Conde K, Erickson SK et al. 1998. Hypolipidemic mechanisms of pectin and psyllium in guinea pigs fed high fat-sucrose diets: alterations on hepatic cholesterol metabolism. J Lipid Res, 39:1455-65.

Walker AR. 1974. Editorial: Dietary fiber and the pattern of diseases. Ann Intern Med, 80:663-4.

Wong J, Quinn CM, Brown AJ. 2006. SREBP-2 positively regulates transcription of the cholesterol efflux gene, ABCA1, by generating oxysterol ligands for LXR. Biochem J, 400:485-91.

Wu JE, Basso F, Shamburek RD, et al. 2004. Hepatic ABCG5 and ABCG8 overexpression increases hepatobiliary sterol transport but does not alter aortic atherosclerosis in transgenic mice. $J$ Biol Chem, 279:22913-25.

Wu K, Bowman R, Welch AA, et al. 2007. Apolipoprotein E polymorphisms, dietary fat and fibre, and serum lipids: the EPIC Norfolk study. Eur Heart J, 28:2930-6.

Yano K, Rhoads GG, Kagan A, et al. 1978. Dietary intake and the risk of coronary heart disease in Japanese men living in Hawaii. Am J Clin Nutr, 31:1270-9.

Yang X, Yang C, Farberman A et al. 2008. The Mammalian target of rapamycin-signaling pathway in regulating metabolism and growth. J Anim Sci, 86(E. Suppl.):E36-E50.

Zannis VI. 1986. Genetic polymorphism in human apolipoprotein E. Methods Enzymol, 128:823-51.

Zhang JX, Hallmans G, Andersson H, et al. 1992. Effect of oat bran on plasma cholesterol and bile acid excretion in nine subjects with ileostomies. Am J Clin Nutr, 56:99-105. 
